\title{
Accountability Assessment of Health Improvements in the United States Associated with Reduced Coal Emissions Between 2005 and 2012
}

\author{
Lucas RF Henneman ${ }^{\mathrm{a}}$, Christine Choirat ${ }^{\mathrm{a}}$, Corwin M Zigler ${ }^{\mathrm{a}}$ \\ aDepartment of Biostatistics, Harvard T.H. Chan School of Public Health, Boston, MA
}

\begin{abstract}
Background: National, state, and local policies contributed to a $65 \%$ reduction in sulfur dioxide emissions from coal-fired power plants between 2005 and 2012 in the United States, providing an opportunity to directly quantify public health benefits attributable to these reductions under an air pollution accountability framework.
\end{abstract}

Methods: We estimate ZIP code-level changes in two different—but related—exposure metrics: total $\mathrm{PM}_{2.5}$ concentrations and exposure to coal-fired power plant emissions. We associate changes in 10 health outcome rates among approximately 30 million US Medicare beneficiaries with exposure changes between 2005 and 2012 using two difference-in-difference regression approaches designed to mitigate observed and unobserved confounding.

Results: Rates per 10,000 person-years of six cardiac and respiratory health outcomes-all cardiovascular disease, chronic obstructive pulmonary disorder, cardiovascular stroke, heart failure, ischemic heart disease, and respiratory tract infections-decreased by between 7.89 and 1.95 per $\mu \mathrm{gm}^{-3}$ decrease in $\mathrm{PM}_{2.5}$, with comparable decreases in coal exposure leading to slightly larger rate decreases. Results for acute myocardial infarction, heart rhythm disorders, and peripheral vascular disease were near zero and/or mixed between the various exposure metrics and analyses. A secondary analysis found that nonlinearities in relationships between changing health outcome rates and coal exposure may explain differences in their associations.

Conclusions: The direct analyses of emissions reductions estimate substantial health benefits via coal power plant emission and $\mathrm{PM}_{2.5}$ concentration reductions. Differing responses associated with changes in the two exposure metrics underscore the importance of isolating source-specific impacts from those due to total $\mathrm{PM}_{2.5}$ exposure.

\section{Keywords}

Air pollution accountability; Coal power plant emissions; Difference-in-difference; $\mathrm{PM}_{2.5}$

Corresponding author: Lucas RF Henneman, 655 Huntington Avenue, Building $2,4^{\text {th }}$ Floor, Boston, MA 02115, lhenneman@gmail.com, +1 (404) 788-2161.

SDC Supplemental digital content is available through direct URL citations in the HTML and PDF versions of this article (www.epidem.com).

Declaration of competing financial interests: The authors declare no competing financial interests.

Data availability: Data sets and code for reproducing the form of the analysis (with simulated Medicare health outcomes data) are available at osf.io/s $8 \mathrm{fd} 3$. 
Air pollution accountability research investigates the efficacy of air pollution regulations by establishing relationships between one or more links along the so-called chain of accountability: regulations, emissions, air quality, exposure/dose, and health effects. ${ }^{1}$ The accountability field expands the scope of traditional studies in the air pollution epidemiology literature that have focused primarily on establishing relationships between air pollution exposures and adverse health outcomes, ${ }^{2-4}$ which represents just one of the links in the full accountability chain. ${ }^{5}$

Many studies have shown that national, state, and local regulatory actions implemented after the passage of the 1990 Clean Air Act Amendments led to emissions decreases and improved air quality in the United States. ${ }^{6-10}$ Such studies have been used in combination with results from separate studies documenting associations between ambient air quality and adverse health outcomes ${ }^{2,3,11}$ to indirectly infer health benefits of regulatory actions ${ }^{7,12-15}$; however, the most informative assessments of environmental policies that span the accountability chain are relatively less prevalent. ${ }^{16}$ Expanding the scope of health-centered accountability studies backwards along the chain to include emissions and regulations allows for the development of more direct evidence of regulatory efficacy, which is important for continuing policy refinements. ${ }^{5}$

We present an accountability assessment of emissions reductions from coal-fueled power plants (alternatively called electricity generating units) in the United States between 2005 and 2012. Power plants were selected as the source of interest because their emissions, known to be linked to harmful ambient pollution, dramatically decreased over the study period primarily in response to regulatory actions taken by the Environmental Protection Agency (EPA). Important actions implemented during this period include the $\mathrm{NO}_{\mathrm{x}}$ Budget Trading Program and associated State Implementation Call, the Clean Air Interstate Rule, the Cross-State Air Pollution Rule, and updates to the National Ambient Air Quality Standards. ${ }^{17-20}$ Although nonregulatory changes such as economic recessions and availability of natural gas played a role in the observed emissions reductions, recent studies have shown that regulatory actions and controls on individual power plants have led to emissions reductions throughout the United States. ${ }^{21,22}$

Our approach leverages a national ZIP code-level Medicare database to compare changes in rates of 10 health outcomes between 2005 and 2012 across a large swath of the country that experienced differential changes in exposure to coal power plant emissions and exposure to air pollution from all sources. The analysis uses nationally measured coal power plant emissions reported to the EPA, nationwide simulated $\mathrm{PM}_{2.5}$ concentrations, a reduced complexity air quality modeling approach for estimating exposure to coal power plant emissions, and a difference-in-difference analysis designed to control for many common types of observed and unobserved confounding factors. ${ }^{23}$

\section{METHODS}

The analysis approach can be summarized in two steps: (1) we estimated 2005 and 2012 ZIP code level air pollution exposure using two metrics: one novel metric for exposure to coal power plant emissions specifically ("coal exposure") and a second measuring ambient $\mathrm{PM}_{2.5}$ 
concentrations ("total exposure") and (2) we related changes in exposures from 2005 to 2012 with changes in health outcomes rates from 2005 to 2012. The two motivating hypotheses are that (1) areas that have experienced the greatest reductions in exposure have seen the largest decreases in adverse health outcome rates and (2) changes in total exposure have had an impact on health outcomes in different ways than changes in exposure to coal emissions specifically.

\section{Total Exposure: Exposure to Air Pollution from All Sources}

As a metric for total exposure attributable to all air pollution sources, we use ZIP codeaveraged annual ambient $\mathrm{PM}_{2.5}$ concentrations. We employed simulated national $\mathrm{PM}_{2.5}$ concentrations estimated by combining GEOS-Chem chemical transport model-simulated $\mathrm{PM}_{2.5}$, satellite-retrieved aerosol optical depth, and observed $\mathrm{PM}_{2.5}$ concentrations originally described by Boys et al.24 Simulated monthly 0.1 longitude $\times 0.1$ latitude $\mathrm{PM}_{2.5}$ concentrations were averaged to annual concentrations and spatially overlayed on US ZIP codes for both 2005 and 2012. We refer to this exposure field as $\mathrm{PM}_{2.5}^{\mathrm{sim}}$.

\section{Coal Exposure: Exposure to Air Influenced by Coal Emissions}

We calculated coal exposure from each of 1036 (1009) electricity generating units comprising 505 (478) facilities operating in the United States in 2005 (2012) at each ZIP code. The method combines data from continuous emissions monitors 25 and other power plant characteristics 26 with HYSPLIT, an air parcel trajectory and dispersion model.27 We used HYSPLIT to simulate transport and dispersion of parcels originating at each power plant at four start times per day for every day in 2005 and 2012 and track the location of the dispersed particles for 10 days. We then processed raw hourly parcel locations and linked them to ZIP codes, and used an emissions-weighted annual measure of parcel count to derive how emissions from each electricity generating unit impacted each ZIP code. We used $\mathrm{SO}_{2}$ emissions to weight the HyADS metric because $\mathrm{SO}_{2}$ reacts in the atmosphere and contributes to increased $\mathrm{PM}_{2.5}$ concentrations; however, other emitted species (many of which are correlated with $\mathrm{SO}_{2}$ ) may lead to adverse health impacts independent of or interactive to those potentially attributable to $\mathrm{SO}_{2}$ and its atmospheric products. We refer to this calculated HYSPLIT average dispersion metric as HyADS; details appear in eAppendix 1; http://links.lww.com/EDE/B529 and Henneman et al.28

Henneman et al28 found high national and regional correlations between HyADS spatial distributions, observed pollutant species, and the output from an observation-adjusted fullcomplexity chemical transport model. Because HyADS measures exposure to sources as opposed to source contribution to ambient concentrations, HyADS is not reported in measurable units (e.g., $\mu \mathrm{gm}^{-3}$ ). However, to aid comparisons between results from the exposure metrics in the primary analysis described below, we rescaled the unit-less HyADS outputs to match the scale of the observation-adjusted chemical transport model distribution. We calculated adjusted HyADS (HyADS ${ }^{a d j}$, with units $\mu \mathrm{gm}^{-3 *}$ ) using region-specific linear regressions against estimates of US-wide coal emission contributions to $\mathrm{PM}_{2.5} .29$ The rescaled HyADS ${ }^{a d j}$ will be useful for comparisons against analyses of $\mathrm{PM}_{2.5}$. 


\section{Medicare, Census, and Meteorology Data}

We aggregated individual-level primary discharge diagnosis hospitalization outcomes for 24.5 (23.8) million fee-for-service Medicare beneficiaries and all-cause mortality outcomes on these and an additional 4.4 (9.6) million managed care beneficiaries in 2005 (2012) to the level of the patient's residential ZIP code (fee-for-service and managed care are two Medicare coverage plans that keep different records). The study area was confined to ZIP codes located east of -110 longitude (colored ZIP codes in Figure 1, east of the Rocky Mountains) because most of the power plants and their exposures exist in this area.

We calculated ZIP code-level hospitalization rates (per 10,000 person-years) in 2005 and 2012 among fee-for-service beneficiaries for each of the following eight conditions (and corresponding International Classification of Disease, Ninth Revision, Clinical Modification [ICD9-CM] codes): acute myocardial infarction (AMI; ICD9-CM 410.xx), chronic obstructive pulmonary disorder (COPD; ICD9-CM 490.xx-492.xx), cardiovascular stroke (ICD9-CM 430.xx-438.xx), heart failure (ICD9-CM 428.xx), heart rhythm disorders (ICD9CM 426.xx-427.xx), ischemic heart disease (IHD; ICD9-CM 410.xx-414.xx and 429.xx; note: includes AMI), peripheral vascular disease (PVD; ICD9-CM 440.xx-448.xx), and respiratory tract infection (RTI; ICD9-CM 464.xx-466.xx and 480.xx-487.xx). A summary of all cardiovascular disease hospitalization (all CVD; 390.xx-459.xx) was also calculated. ZIP code-level all-cause mortality rates (per 10,000 beneficiaries) in 2005 and 2012 were calculated among fee for service and managed care beneficiaries. We selected these outcomes a priori as a representative list of outcomes that have been previously associated with air pollution levels and are available in Medicare hospitalization data. The use of administrative billing data has potential limitations for classifying complex diseases.

Census data were extrapolated by Esri Business Analyst Demographic Data (Esri, Redlands, California). We used variables for median age, median household income, per-capita income, gender, and race. The data were available from the year 2007 and later; therefore, 2007 was used as a proxy for 2005 . The fraction of population by county that smoked in 2000 was available from previously-produced small-area estimates derived from Center for Diseases Control and Prevention Behavioral Risk Factor Surveillance System data. ${ }^{30}$ Temperature and specific humidity were downloaded from the North American Regional Reanalysis and spatially aggregated to ZIP codes. ${ }^{31}$

\section{Statistical Analysis: Difference-in-difference Regression}

To associate changes in each health outcome rate with changes in exposure, we use a difference-in-difference regression approach, which is designed to mitigate certain types of observed and unobserved confounding. ${ }^{23}$ The models take the form:

$$
R_{2012-2005}^{\Delta}=\alpha_{0}+\beta_{E}^{\Delta} E_{2012-2005}^{\Delta}+\sum_{c=1}^{C} \beta_{c} X_{c, 2005}+\sum_{c=1}^{C} \beta_{c c, 2012-2005}^{C}+\varepsilon
$$

where $R_{2012-2005}^{\Delta}$ represents the differences between 2012 and 2005 health outcome rates (per 10,000 person-years for hospitalization outcomes, per 10,000 beneficiaries for 
mortality) and $E_{2012-2005}^{\Delta}$ represents the differences between 2012 and 2005 exposures characterized by one of the two methods described above. Census and meteorology covariates listed in eTable 1 are represented with $X_{c, 2005}$ and changes in covariate values from 2005 to 2012 are represented with $X_{c, 2012-2005}^{\Delta}$. All differences (2012-2005) are taken as the 2012 value minus the 2005 value, so decreases over this time span are negative values. Base year values and changes over time are both included in the model to account for ZIP codes that are different at the beginning of the study period (e.g., warmer vs. cooler areas) and areas that may have changed differently between 2005 and 2012 (e.g., increases vs. decreases in temperature). $\varepsilon$ represents a random error term, assumed to follow a Gaussian distribution with mean zero.

The model in equation 1 implies two important simplifications. First, the regression coefficients $\left(\beta_{E}^{\Delta} \mathrm{s}\right)$ represent the linear association between the changes in health outcome rate and changes in exposure across the entire study population, and cannot provide a detailed description of possible heterogeneity in health responses across varying degrees of exposure change. Second, comparisons of results across different exposure metrics $(E)$ with the model in equation 1 relies on the aforementioned rescaling of the unit-less HyADS metric to compare against total $\mathrm{PM}_{2.5}^{\mathrm{sim}}$ exposure measured in $\mu \mathrm{gm}^{-3}$. To investigate nonlinearities and avoid rescaling HyADS, we apply a secondary approach that estimates responses at varying quantiles of exposure change:

$$
R_{2012-2005}^{\Delta}=\sum_{q=1}^{M} \beta_{q} Q_{q}+\sum_{c=1}^{C} \beta_{c} X_{c, 2005}+\sum_{c=1}^{C} \beta_{c}^{\Delta} X_{c, 2012-2005}^{\Delta}+\varepsilon
$$

where all notation is as in equation 1 , with the key difference that $\mathrm{Q}_{q}, q=1,2, \ldots, M$ is a categorical variable that represents membership in one of the $M$ quantiles of the distribution of exposure reductions; $Q_{1}=1$ for all ZIP codes with the smallest exposure reductions (which may in fact be exposure increases), and $Q_{M}=1$ for ZIP codes with the largest reductions. The coefficients $\beta_{q}$ are akin to separate intercepts for each quantile of the exposure reduction distribution, with quantities such as $\beta_{q}-\beta_{q^{\prime}}$ representing the estimated difference in health outcome rate change between areas with exposure reductions in two different quantiles of the distribution (adjusting for covariates). If the hypothesis that larger decreases in exposure leads to greater decreases in health outcomes holds, the values of $\beta_{q}$ are expected to increase in $q$. In this modeling, we specify a priori $M=20$ quantiles, each of which is called a ventile.

We perform two statistical tests to establish whether (1) health improved more at higher levels of exposure decrease across the distribution of quantiles (i.e., the estimates of $\beta_{q}$ increase in $q$ ) and (2) the health improvement across changes in exposure quantiles is linear (i.e., $\beta_{q}-\beta_{q+1}$ is the same for $\left.q=1,2, \ldots, 19\right)$. These tests employ a slight reformulation of the model in equation 2 to specify a linear trend in $Q_{q}$ : 


$$
R_{2012-2005}^{\Delta}=\alpha_{0}+\beta_{q}^{*} Q_{q}+\sum_{c=1}^{C} \beta_{c} X_{c, 2005}+\sum_{c=1}^{C} \beta_{c}^{\Delta} X_{c, 2012-2005}^{\Delta}+\varepsilon
$$

where $\beta_{q}^{*}$ represents the linear slope in $R_{2012-2005}^{\Delta}$ with increasing ventile $q$, a standard significance test determines whether the linear trend $\beta_{q}^{*}$ is statistically different from zero. A likelihood ratio test on the differences in model fits between model 3 and model 2 provides a formal summary of whether the relationship between $R_{2012-2005}^{\Delta}$ and $Q_{q}$ is nonlinear. Model 3 is only used as a basis for the two statistical tests pertaining linear trends, and health impact estimates are not reported from this model.

\section{RESULTS}

On average, annual $\mathrm{PM}_{2.5}^{\text {sim }}$ concentrations decreased by $3.2 \mu \mathrm{gm}^{-3}(32 \%)$ while HyADS coal exposure decreased by $69 \%$ (Table 1 ), similar to the $65 \%$ decrease in coal power plant $\mathrm{SO}_{2}$ emissions. Geographic patterns of exposure reductions in both metrics were similarconcentrated in the eastern United States—but not identical (Figure 1). PM ${ }_{2.5}^{\text {sim }}$ and HyADS simulated exposure increases in a few ZIP codes, confined in both cases to the first ventile of the distribution of exposure decreases ( $Q_{1}$; eFigure 1; http://links.lww.com/EDE/B529). The largest decreases in the exposure metrics generally follow two distinct bands, one along the Ohio River Valley and the other extending southeast from Pennsylvania through the Southeastern United States. Annual values of HyADS ${ }^{a d j}$ as a percentage of total PM ${ }_{2.5}^{\text {sim }}$ agree with previously-published estimates. ${ }^{32,33}$

Rates of all 10 health outcomes decreased on average across all ZIP codes, although variability across ZIP codes was large-interquartile ranges of the ZIP code differences of all ten outcomes indicate increased rates in some areas (Table 2). Changes in other covariates were mixed (eTable 1); most saw small changes in their mean value between 2005 and 2012.

\section{Primary Analysis: Adjusted Linear Associations}

Associations estimated with model 1 plotted in Figure 2 and reported in eTable 2 represent the change in health outcome rate (per 10,000 person-years [beneficiaries] for hospitalizations [mortality]) per unit change in each exposure metric. Recall that exposure changes for models 1 and 2 are calculated so that decreases from 2005 to 2012 are negative vales. Throughout this work we report estimates of $-\beta_{E}^{\Delta}$ and $-\beta_{q}$ to highlight changes in health outcomes rates associated with exposure reductions.

Reductions in rates of all CVD, cardiovascular stroke, heart failure, IHD, and RTI between 2005 and 2012 are associated with decreases in both measures of exposure- $\mathrm{PM}_{2.5}^{\mathrm{sim}}$ and HyADS $^{a d j}$. For these five outcomes, health outcome rate decreases per $\mu \mathrm{gm}^{-3}$ decrease in 
$\mathrm{PM}_{2.5}^{\text {sim }}$ range between 1.95 and 8.40, and decreases per unit $\mu \mathrm{gm}^{-3 *}$ decrease in HyADS ${ }^{a d j}$ range between 4.24 and 26.11. Health outcome rate decreases tend to be more pronounced for HyADS ${ }^{a d j}$, particularly for all CVD and heart failure. The point estimates of changes in hospitalization rate per 10,000 person-years per unit decrease in each exposure metric $\left(-\beta_{E}^{\Delta}\right)$ for all CVD differ by more than a factor of three for $\mathrm{PM}_{2.5}^{\mathrm{sim}}(-8.40$, confidence interval [CI] $=-12.67,-4.14)$ and $\operatorname{HyADS}^{a d j}(-26.11, \mathrm{CI}=-33.89,-18.33)$.

Changes in AMI and COPD rates are not associated with changes in total exposure, whereas reductions in HyADS ${ }^{a d j}$ coal exposure is associated with reduced hospitalization rates for AMI $(-2.29, \mathrm{CI}=-3.91,-0.66)$ and $\operatorname{COPD}(-5.19, \mathrm{CI}=-8.13,-2.25)$.

Increases in the rate of heart rhythm disorders are marginally associated with decreases in $\mathrm{PM}_{2.5}^{\operatorname{sim}}(0.96, \mathrm{CI}=-0.21,2.12)$ and $\operatorname{HyADS}^{\mathrm{adj}}(0.95, \mathrm{CI}=-1.14,3.05)$ exposure. Changes in PVD and mortality are not associated with either of the exposure metrics.

For comparison, a version of model 1 without the adjustment for observed covariates provided similar results (eFigure 2), indicating that the observed covariates had little impact on the difference-in-difference analysis. A notable exception occurs in unadjusted analysis of all cause Mortality, where associations are negative for both $\mathrm{PM}_{2.5}^{\mathrm{sim}}$ and HyADS, contrary to the null findings in the adjusted model. eAppendix 2; http://links.lww.com/EDE/B529 describes the significant adjustment covariates in the models for mortality.

\section{Secondary Analysis: Adjusted Quantile Associations}

The motivating hypotheses can be assessed by comparing the estimates of $\beta_{q}$ for different values of $q$; the quantity $\beta_{q}-\beta_{q^{\prime}}$ for $q \neq q^{\prime}$ represents the difference in health reduction for two different quantiles of exposure decrease (adjusting for covariates). Figure 3 provides a visual means to compare estimates of all $\beta_{q}$, with negative values denoting improved health at higher ventiles of exposure decrease. eTable 3 provides numerical comparisons for selected ventiles.

While estimates of $\beta_{q}$ 's plotted in Figure 3 are not modeled to have any particular trend, linear trends in the estimated $-\beta_{q}$ 's have been superimposed to highlight whether the trend is increasing, decreasing, or flat. $P$-values from formal statistical tests of whether estimates of $-\beta_{q}$ show a decreasing trend in $q$ are derived from model 3 and reported as $P_{\beta_{q}^{*}}$ in eTable 4.

Likelihood ratio tests of whether the flexible specification in model 2 provides a better fit to the data than the linear form of model 3 , presented in eTable 4 , indicate adequacy of the linear fit to quantiles $\left(P_{l i n}>0.05\right)$ for eight out of $10 \mathrm{PM}_{2.5}^{\text {sim }}$ models (all except heart failure and IHD) but only one HyADS model (all Mortality). This indicates more nonlinearity in the relationship between decreasing HyADS exposure and changes in health outcomes, which is potentially a result of the $\mathrm{PM}_{2.5}^{\text {sim }}$ surface that is much more evenly spaced than the HyADS (eFigure 1). 
Consistent with the results of the primary analysis, all CVD, cardiovascular stroke, heart failure, IHD, and RTI rates all show more pronounced health improvements at ventiles representing greater exposure reductions (Figure 3), and linear hypothesis tests suggest that the trends in $-\beta_{q}$ for each of these outcomes are different from zero $\left(P_{\beta_{q}^{*}}<0.05\right.$; eTable 4; http://links.lww.com/EDE/B529). The slope in $-\beta_{q}$ for the PM ${ }_{2.5}^{\text {sim }}$ all CVD model plotted in Figure 3 is steeper than that for the HyADS model, seemingly at odds with the finding in the primary analysis that the slope between changing all CVD and changing HyADS ${ }^{a d j}$ exposure is a factor of three greater than that for $\mathrm{PM}_{2.5}^{\mathrm{sim}}$. However, $\beta_{q}$ in the HyADS all CVD model is not linear in $q$, as evidenced by the scatter in Figure 3 and $P_{l i n}<10^{-16}$. Further, comparisons between differences in HyADS $\beta_{q}$ 's are highly variable, ranging from $\beta_{5}-\beta_{10}=-52.8$ to $\beta_{10}-\beta_{15}=22.7$. High variability in the change in all CVD rate per 10,000 across ventiles of exposure change provides a potential explanation for the large difference between the $\mathrm{PM}_{2.5}^{\mathrm{sim}}$ and HyADS models in the primarily analysis.

AMI, PVD, and all-cause mortality show small or slightly negative linear trends between responses and increasing exposure reductions, again similar across exposure metrics for each health outcome. Hypothesis tests of the linear trend in AMI, PVD, and mortality find that we cannot reject the null hypothesis that the slope in the trends is equal to zero $\left(P_{\beta_{q}^{*}}>0.05\right.$;

eTable 4). The rate of heart rhythm disorders rate changes again decreased with larger exposure reductions (evidenced by the positive slopes in Figure 3). Differences in $-\beta_{q}$ plotted in Figure 3 and summarized in eTable 4 suggest that the apparent adverse effect of reduced air pollution exposure is confined to areas that experienced the smallest exposure reductions.

In the secondary analysis, COPD again shows minimal change associated with changing total exposure, and a statistically significant negative change in rate associated with each HyADS ventile $\left(P_{\beta_{q}^{*}}<0.05\right)$. Higher nonlinearity in the HyADS association is apparent in trends in $\beta_{q}$ 's plotted in Figure 3 and summarized in eTable 3 (http://links.lww.com/EDE/ B529) and as $P_{\text {lin }}<10^{-4}$.

\section{DISCUSSION}

This study investigates the association between health improvements among US Medicare beneficiaries and concurrent changes in emissions from coal-fired power plants and total $\mathrm{PM}_{2.5}$ exposure from 2005 to 2012 . The combination of tools developed by the atmospheric modeling community (i.e., $\mathrm{PM}_{2.5}^{\mathrm{sim}}$ and HyADS) with statistical methods anchored to causal and quasi-experimental approaches span the accountability chain from Emissions to Health Effects.

We explore three important aspects of this study in depth: the results, the approach, and the relevance within the existing body of air pollution accountability literature. 


\section{Reducing Exposure and Adverse Health Outcomes}

The 10 health outcomes can be organized into four groups based on results of the primary and secondary analyses. In the first group - consisting of all CVD, cardiovascular stroke, heart failure, IHD, and RTIs - rate reductions are associated with reductions in all three exposure metrics at statistically significant levels in both analyses. The results provide strong evidence that reduced exposure to total $\mathrm{PM}_{2.5}$ and coal emissions have led to reduced rates of these outcomes, agreeing with previous findings of at minimum suggestive evidence that incidences of each are associated with long-term exposure to $\mathrm{PM}_{2.5}$ and/or other pollutants (all CVD, ${ }^{34,35}$ cardiovascular stroke, ${ }^{36}$ heart failure, ${ }^{37} \mathrm{IHD},{ }^{37,38}$ and $\mathrm{RTIs}^{34}$ ).

The second group is comprised of AMI and heart rhythm disorders, changes of which are characterized by weak (AMI) and positive (heart rhythm disorders) associations with exposure reductions and ambiguity between results in the primary and secondary analyses. AMI rate reductions, which are marginally associated with coal exposure reductions in the primary analysis, have no clear trend in association with either of the exposure metrics in the secondary analysis (i.e., trends in $-\beta_{q}$ are not significantly different than zero). AMI is an acute affliction that has been associated with short-duration (hourly and daily) spikes in $\mathrm{PM}_{2.5}$ concentrations. ${ }^{39}$ Ostro et al ${ }^{35}$ did not find associations between AMI and a subset of heart rhythm disorders (ICD9 codes 427.xx) with total multi-year average $\mathrm{PM}_{2.5}$, but did find positive associations between both outcomes and $\mathrm{PM}_{2.5}$ from other sources (especially vehicular emissions), highlighting that these important sources related to these outcomes are not captured in the present analysis.

In the third group, PVD and all-cause mortality rate changes have very small associations with reduced exposures. While meta-analysis estimates by Hoek et $\mathrm{al}^{40}$ and others have found excess risk of all-cause mortality and mortality from CVD associated with increased air pollution concentrations, our results suggest that mean effects of exposure reductions have little effect on changes in all-cause mortality. Hoffmann et $\mathrm{al}^{41}$ found increased risk of peripheral arterial disease (related to PVD) associated with distance between study participants' home addresses and major roads, but inconsistent results with increasing $\mathrm{PM}_{2.5}$ concentration. The PM ${ }_{2.5}^{\text {sim }}$ modeling used here (and the one used by Hoffmann et $\mathrm{al}^{41}$ ) likely is not of high enough resolution to completely capture $\mathrm{PM}_{2.5}$ composition and concentration spatial distributions around roads. The current study finds little evidence of decreased PVD incidence that have accompanied coal emissions reductions.

Previous studies using individual-level data have identified associations between mortality and ambient $\mathrm{PM}_{2.5}$ concentrations. ${ }^{2-4}$ An important difference between the present analysis and previous analyses documenting a $\mathrm{PM}_{2.5}$-mortality association is the present reliance on aggregated (to the level of ZIP code) data. Previous studies of individual-level data are particularly suited to the study of a more diffuse signal such as that relating ambient $\mathrm{PM}_{2.5}$ and mortality, which is expected to be more diffuse than the signal relating to hospitalizations.

The final group consists only of COPD rate, changes of which show little association with $\mathrm{PM}_{2.5}^{\mathrm{sim}}$ exposure changes in either the primary or secondary analyses. Decreases in HyADS 
coal exposure, however, are strongly associated with decreases in COPD rate in both analyses. Together, these results suggest a potential enhanced benefit of COPD hospitalization reductions in areas with greater coal exposure reductions. Previous evidence supports an outsize influence of annual average $\mathrm{SO}_{2}$ exposure on COPD diagnoses over total $\mathrm{PM}_{2.5}$ exposure ${ }^{42}$ and short-term exposure to secondary nitrate (a $\mathrm{PM}_{2.5}$ constituent formed in the atmosphere as a byproduct of fossil fuel emissions) on COPD rates. ${ }^{35}$

\section{Approach}

Insofar as the HyADS approach to model coal-specific exposure entails some simplifications relative to full-scale chemical transport models, it gains functionality important to the present analysis. For instance, the ability to identify impacts from individual units on a national scale is an improvement over related studies that employ source apportionment techniques to separate observed $\mathrm{PM}_{2.5}$ concentrations into contributions from various source categories, ${ }^{35,38}$ and the computational convenience to explore individual units across multiple time points improves on studies anchored to full-scale chemical transport models. 43,44

The difference-in-difference study design and analysis approach, sometimes described as a "quasi-experimental" method, has been leveraged in many research domains, including environmental accountability studies. ${ }^{23,45-47}$ The primary benefit of this approach is its potential to mitigate the threat of confounding due to two types of measured or unmeasured factors: (1) differing characteristics between ZIP codes that are associated with exposure levels and health outcomes are "canceled out" when calculating differences over time within ZIP codes; (2) comparing these differences across ZIP codes similarly "cancels out" temporal trends common across all ZIP codes.

Nonetheless, as with any statistical analysis of observational data, the threat of confounding cannot be definitively ruled out. For example, the difference-in-difference analysis does not adjust for unobserved factors that differentially change across areas in a way that is related to changes in exposure and health. One example, in this case, would be if EPA's designations of non-attainment with the National Ambient Air Quality Standards initiated emissions reductions during this time frame but also triggered economic or population shifts that manifest as reductions in mortality and hospitalization rates in locations where emissions decreased the most. The present analysis adjusted the difference-in-difference analysis for a variety of location-specific demographic characteristics, and temperature and specific humidity to protect against these threats to validity, but the possibility of unmeasured confounding factors that change differentially over time across areas remains.

The approach assumes that any exposure measurement error is similar across the HyADS and $\mathrm{PM}{ }_{2.5}^{\text {sim }}$ metrics. While a direct comparison of error between the two approaches is difficult to implement, the secondary analysis based on quantiles should prove particularly robust to exposure measurement error. Spatial autocorrelation is addressed through the inclusion of spatially autocorrelated predictors in the regression, but models that account for any residual spatial autocorrelation could be of interest in future analyses. 


\section{Air Pollution Accountability Implications}

This work adds to the growing body of evidence that US air quality regulations and policies implemented across many years over large spatial domains have improved both air quality and health..$^{6,8,21,48,49}$ The direct analyses of emissions reductions provide evidence consistent with widespread benefits achieved through total $\mathrm{PM}_{2.5}$ exposure reductions and coal power plant emission reductions over a 7-year period.

The Health Effects Institute (2003) and Zigler and Dominici (2014) argued that future regulatory actions can particularly benefit from direct evidence of the effectiveness of specific policies or emissions reductions, as opposed to indirect evidence from traditional epidemiologic studies that establish associations between health outcomes and air pollution. 1,5 Importantly in this regard, we estimate impacts of emissions reductions on health outcomes without relying on historic estimates of concentration-response relationships, as is common in previous studies. ${ }^{50,51}$ Both the adaptation of the difference-in-difference approach and the extension of the project scope backwards along the accountability chain to link emissions to health response leads to an assessment closer to that of a direct analysis of a specific action or intervention that initiates the accountability chain.

\section{Supplementary Material}

Refer to Web version on PubMed Central for supplementary material.

\section{Sources of financial support:}

This work was supported by research funding from NIHR01ES026217, EPA 83587201, and HEI 4953. Its contents are solely the responsibility of the grantee and do not necessarily represent the official views of the USEPA. Further, USEPA does not endorse the purchase of any commercial products or services mentioned in the publication.

\section{References}

1. Health Effects Institute. Assessing Health Impact of Air Quality Regulations: Concepts and Methods for Accountability Research. Technical Report. 2003.

2. Dockery DW, Pope CA, Xu X, et al. An association between air pollution and mortality in six U.S. cities. N Engl J Med. 1993;329:1753-1759. [PubMed: 8179653]

3. Laden F, Schwartz J, Speizer FE, Dockery DW. Reduction in fine particulate air pollution and mortality: extended follow-up of the Harvard Six Cities study. Am J Respir Crit Care Med. 2006;173:667-672. [PubMed: 16424447]

4. Di Q, Wang Y, Zanobetti A. Air pollution and mortality in the Medicare population. N Engl J Med. 2017;376:2513-2522. [PubMed: 28657878]

5. Zigler CM, Dominici F. Point: clarifying policy evidence with potential-outcomes thinking-beyond exposure-response estimation in air pollution epidemiology. Am J Epidemiol. 2014;180:1133-1140. [PubMed: 25399414]

6. Henneman LRF, Chang HH, Liao KJ, Lavoué D, Mulholland JA, Russell AG. Accountability assessment of regulatory impacts on ozone and PM2.5 concentrations using statistical and deterministic pollutant sensitivities. Air Qual Atmos Health. 2017;10:695-711.

7. United States Environmental Protection Agency. The Benefits and Costs of the Clean Air Act from 1990 to 2020 Final Report. Technical Report. 2011.

8. Harrington W, Morgenstern R, Shih J, Bell ML. Did the clean air act amendments of 1990 really improve air quality? Air Qual Atmos Health. 2012;5:353-367. 
9. Gégo E, Gilliland A, Godowitch J, Rao ST, Porter PS, Hogrefe C. Modeling analyses of the effects of changes in nitrogen oxides emissions from the electric power sector on ozone levels in the eastern United States. J Air Waste Manag Assoc. 2008;58:580-588. [PubMed: 18422044]

10. Daskalakis N, Tsigaridis K, Myriokefalitakis S, Fanourgakis GS, Kanakidou M. Large gain in air quality compared to an alternative anthropogenic emissions scenario. Atmos Chem Phys. 2016;16:9771-9784.

11. Krewski D, Jerrett M, Burnett RT, et al. Extended follow-up and spatial analysis of the American Cancer Society study linking particulate air pollution and mortality. Res Rep Health Eff Inst. 2009:5-114; discussion 115-36,.

12. Pappin AJ, Mesbah SM, Hakami A, Schott S. Diminishing returns or compounding benefits of air pollution control? The case of NOx and ozone. Environ Sci Technol. 2015;49:9548-9556. [PubMed: 26207850]

13. Dedoussi I, Barrett S. Air pollution and early deaths in the United States. Part II: attribution of PM2.5 exposure to emissions species, time, location and sector. Atmos Environ. 2014;99:610-617.

14. Fann N, Baker KR, Fulcher CM. Characterizing the PM2.5-related health benefits of emission reductions for 17 industrial, area and mobile emission sectors across the U.S. Environ Int. 2012;49:141-151. [PubMed: 23022875]

15. Siler-evans K, Lima I, Morgan MG, Apt J. Regional variations in the health, environmental, and climate benefits of wind and solar generation. Proc Natl Acad Sci U S A. 2013;110:11768-11773. [PubMed: 23798431]

16. Henneman LR, Liu C, Mulholland JA, Russell AG. Evaluating the effectiveness of air quality regulations: a review of accountability studies and frameworks. J Air Waste Manag Assoc. 2016;67:144-172.

17. United States Environmental Protection Agency. Regulatory Impact Analysis for the NBP. Technical Report. 1998.

18. United States Environmental Protection Agency. Acid Rain Program 2001 Summary Report. Technical Report. 2002.

19. United States Environmental Protection Agency. Regulatory Impact Analysis for the Final Clean Air Interstate Rule. Technical Report, Office of Air and Radiation. 2005.

20. United States Environmental Protection Agency. National Ambient Air Quality Standards for Ozone Final Rule. 2015.

21. Russell AG, Tolbert PE, Henneman LRF, et al.; Health Effects Instititute. Impacts of Regulations on Air Quality and Emergency Department Visits in the Atlanta Metropolitan Area. Technical Report 195. 2018.

22. Zigler CM, Kim C, Choirat C, et al.; Health Effects Instititute. Causal Inference Methods for Estimating Long-Term Health Effects of Air Quality Regulations. Technical Report 187. 2016.

23. Greenstone M, Gayer T. Quasi-experimental and experimental approaches to environmental economics. J Environ Econ Manage. 2009;57:21-44.

24. Boys BL, Martin RV, van Donkelaar A, et al. Fifteen-year global time series of satellite-derived fine particulate matter. Environ Sci Technol. 2014;48:11109-11118. [PubMed: 25184953]

25. United States Environmental Protection Agency. Air Markets Program Data. 2016.

26. United States Environmental Protection Agency. National Emissions Inventory. Technical Report. 2016.

27. Stein AF, Draxler RR, Rolph GD, Stunder BJB, Cohen MD, Ngan F. NOAA's HYSPLIT atmospheric transport and dispersion modeling system. Bull Am Meteorol Soc. 2015;96:20592077.

28. Henneman LRF, Choirat C, Ivey C, Cummiskey K, Zigler CM. Characterizing population exposure to coal emissions sources in the United States using the HyADS model. Atmos Environ. 2019;203:271-280.

29. Ivey CE, Holmes HA, Hu YT, Mulholland JA, Russell AG. Development of PM2.5 source impact spatial fields using a hybrid source apportionment air quality model. Geosci Model Dev. 2015;8:2153-2165. 
30. Dwyer-Lindgren L, Mokdad AH, Srebotnjak T, Flaxman AD, Hansen GM, Murray CJ. Cigarette smoking prevalence in US counties: 1996-2012. Popul Health Metr. 2014;12:5. [PubMed: 24661401]

31. Kalnay E, Kanamitsu M, Kistler R, et al. The NCEP/NCAR 40-year reanalysis project. Bull Am Meteorol Soc. 1996;77:437-471.

32. Thurston GD, Ito K, Lall R. A source apportionment of US fine particulate matter air pollution. Atmos Environ (1994). 2011;45:3924-3936. [PubMed: 24634604]

33. Blanchard CL, Tanenbaum S, Hidy GM. Source contributions to atmospheric gases and particulate matter in the southeastern United States. Environ Sci Technol. 2012;46:5479-5488. [PubMed: 22475316]

34. Tolbert PE, Klein M, Peel JL, Sarnat SE, Sarnat JA. Multipollutant modeling issues in a study of ambient air quality and emergency department visits in Atlanta. J Expo Sci Environ Epidemiol 2007;17(2 suppl):S29-S35. [PubMed: 18079762]

35. Ostro B, Malig B, Hasheminassab S, Berger K, Chang E, Sioutas C. Associations of sourcespecific fine particulate matter with emergency department visits in California. Am J Epidemiol. 2016;184:450-459. [PubMed: 27605585]

36. Stafoggia M, Cesaroni G, Peters A, et al. Long-term exposure to ambient air pollution and incidence of cerebrovascular events: results from 11 European cohorts within the ESCAPE project. Environ Health Perspect. 2014;122:919-925. [PubMed: 24835336]

37. Pope CA III, Burnett RT, Thurston GD, et al. Cardiovascular mortality and long-term exposure to particulate air pollution: epidemiological evidence of general pathophysiological pathways of disease. Circulation. 2004;109:71-77. [PubMed: 14676145]

38. Thurston GD, Burnett RT, Turner MC, et al. Ischemic heart disease mortality and long-term exposure to source-related components of U.S. fine particle air pollution. Environ Health Perspect. 2016;124:785-794. [PubMed: 26629599]

39. Peters A, Dockery DW, Muller JE, Mittleman MA. Increased particulate air pollution and the triggering of myocardial infarction. Circulation. 2001;103:2810-2815. [PubMed: 11401937]

40. Hoek G, Krishnan RM, Beelen R, et al. Long-term air pollution exposure and cardio-respiratory mortality: a review. Environ Health. 2013;12:43. [PubMed: 23714370]

41. Hoffmann B, Moebus S, Kröger K, et al. Residential exposure to urban air pollution, ankle-brachial index, and peripheral arterial disease. Epidemiology. 2009;20:280-288. [PubMed: 19194299]

42. Atkinson RW, Carey IM, Kent AJ, van Staa TP, Anderson HR, Cook DG. Long-term exposure to outdoor air pollution and the incidence of chronic obstructive pulmonary disease in a national English cohort. Occup Environ Med. 2015;72:42-48. [PubMed: 25146191]

43. Buonocore JJ, Dong X, Spengler JD, Fu JS, Levy JI. Using the Community Multiscale Air Quality (CMAQ) model to estimate public health impacts of PM2.5 from individual power plants. Environ Int. 2014;68:200-208. [PubMed: 24769126]

44. Levy JI, Baxter LK, Schwartz J. Uncertainty and variability in health-related damages from coalfired power plants in the United States. Risk Anal. 2009;29:1000-1014. [PubMed: 19392676]

45. Pope CA III, Ezzati M, Dockery DW. Fine-particulate air pollution and life expectancy in the United States. N Engl J Med. 2009;360:376-86. [PubMed: 19164188]

46. Correia AW, Pope CA III, Dockery DW, Wang Y, Ezzati M, Dominici F. Effect of air pollution control on life expectancy in the United States: an analysis of 545 U.S. counties for the period from 2000 to 2007. Epidemiology. 2013;24:23-31. [PubMed: 23211349]

47. Greenstone M, Hanna R. Environmental regulations, air and water pollution, and infant mortality in India. Am Econ Rev. 2014;104:3038-3072.

48. Gauderman WJ, Urman R, Avol E, et al. Association of improved air quality with lung development in children. N Engl J Med. 2015;372:905-913. [PubMed: 25738666]

49. Zigler CM, Choirat C, Dominici F. Impact of National Ambient Air Quality Standards nonattainment designations on particulate pollution and health. Epidemiology. 2018;29:165-174. [PubMed: 29095246]

50. U.S. Environmental Protection Agency (EPA). MOtor Vehicle Emissions Simulator (MOVES). 2012. 
51. Fann N, Kim SY, Olives C, Sheppard L. Estimated changes in life expectancy and adult mortality resulting from declining PM2.5 exposures in the contiguous United States: 1980-2010. Environ Health Perspect. 2017;125:097003. [PubMed: 28934094] 

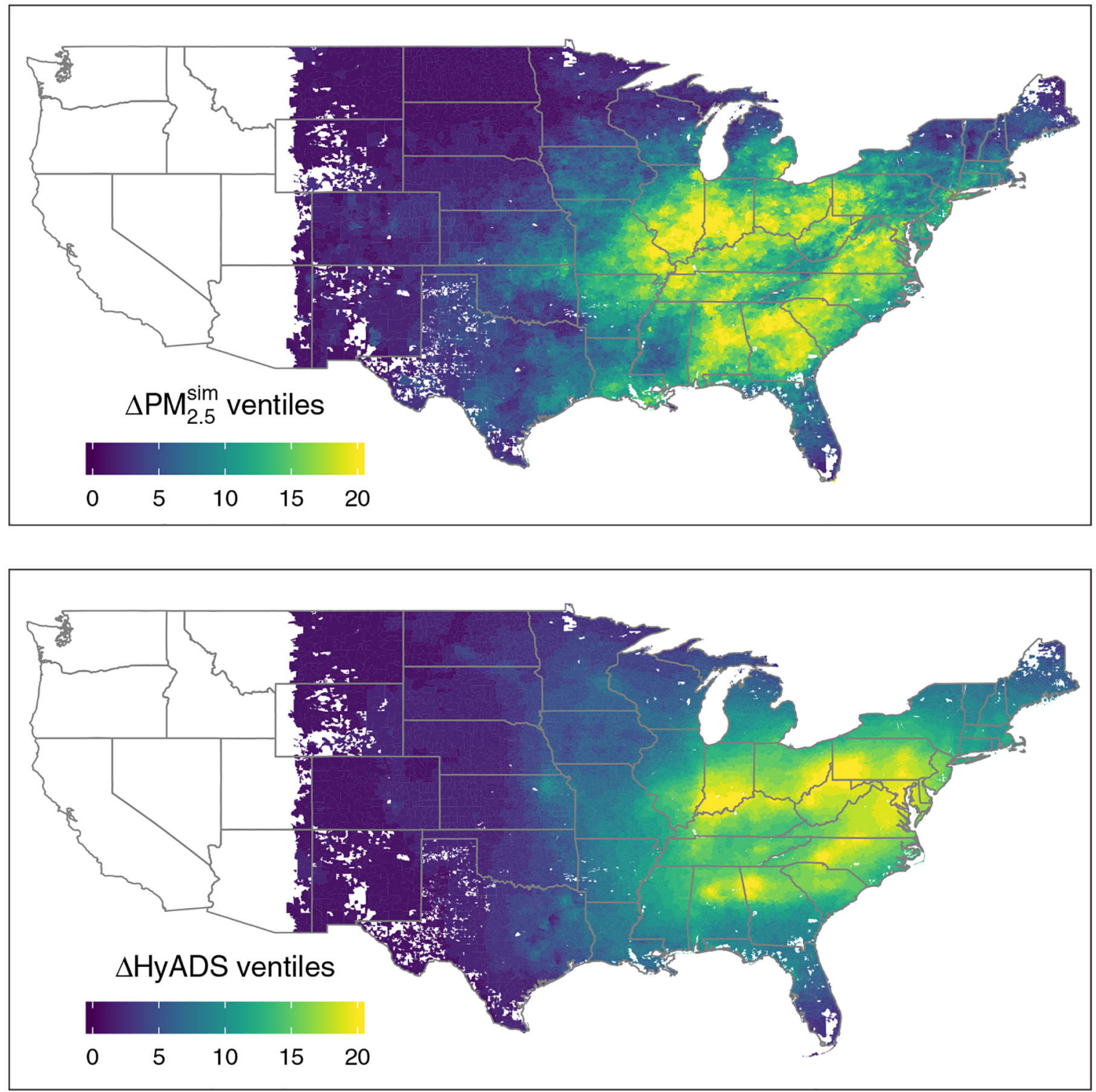

FIGURE 1.

ZIP codes east of -110 longitude colored by ventile of change between 2005 and 2012 (white regions denote excluded areas and areas without ZIP codes). Higher numbered quantiles denote larger decreases. For ventile relationships with each unit's scales, see eFigure 1. Top- $\mathrm{PM}{ }_{2.5}^{\text {sim }}$ exposure, bottom-HyADS coal exposure. 


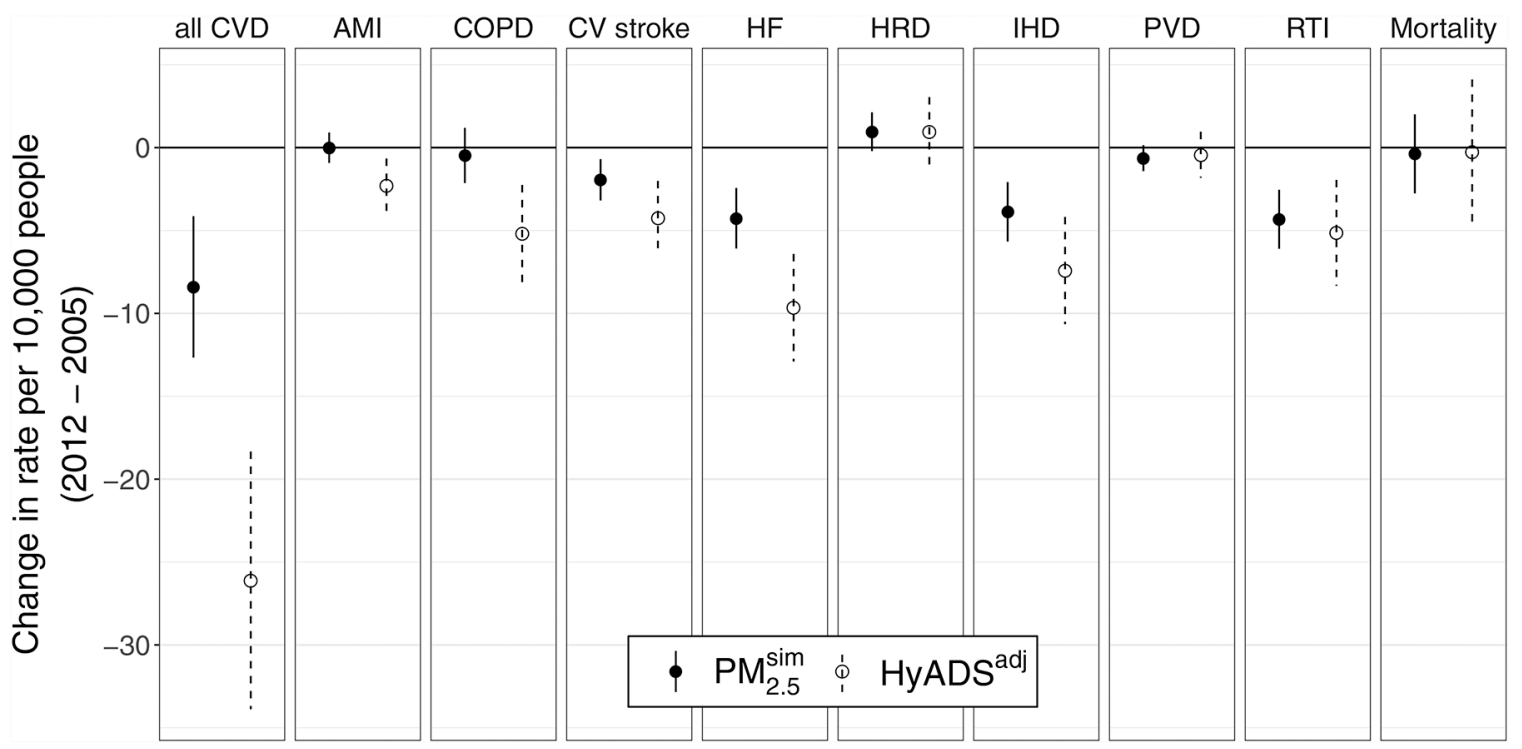

FIGURE 2.

Changes in hospitalization rate per 10,000 person-years (beneficiaries for all Mortality) per unit decrease in each exposure metric $\left(-\beta_{E}^{\Delta}\right)$ estimated with model 1. Negative values on the vertical axis denote decreases in health outcome rates associated with single unit decreases in exposure metrics. Error bars denote the $95 \%$ CIs. CV, cardiovascular; HF, heart failure; HRD, heart rhythm disorders. 


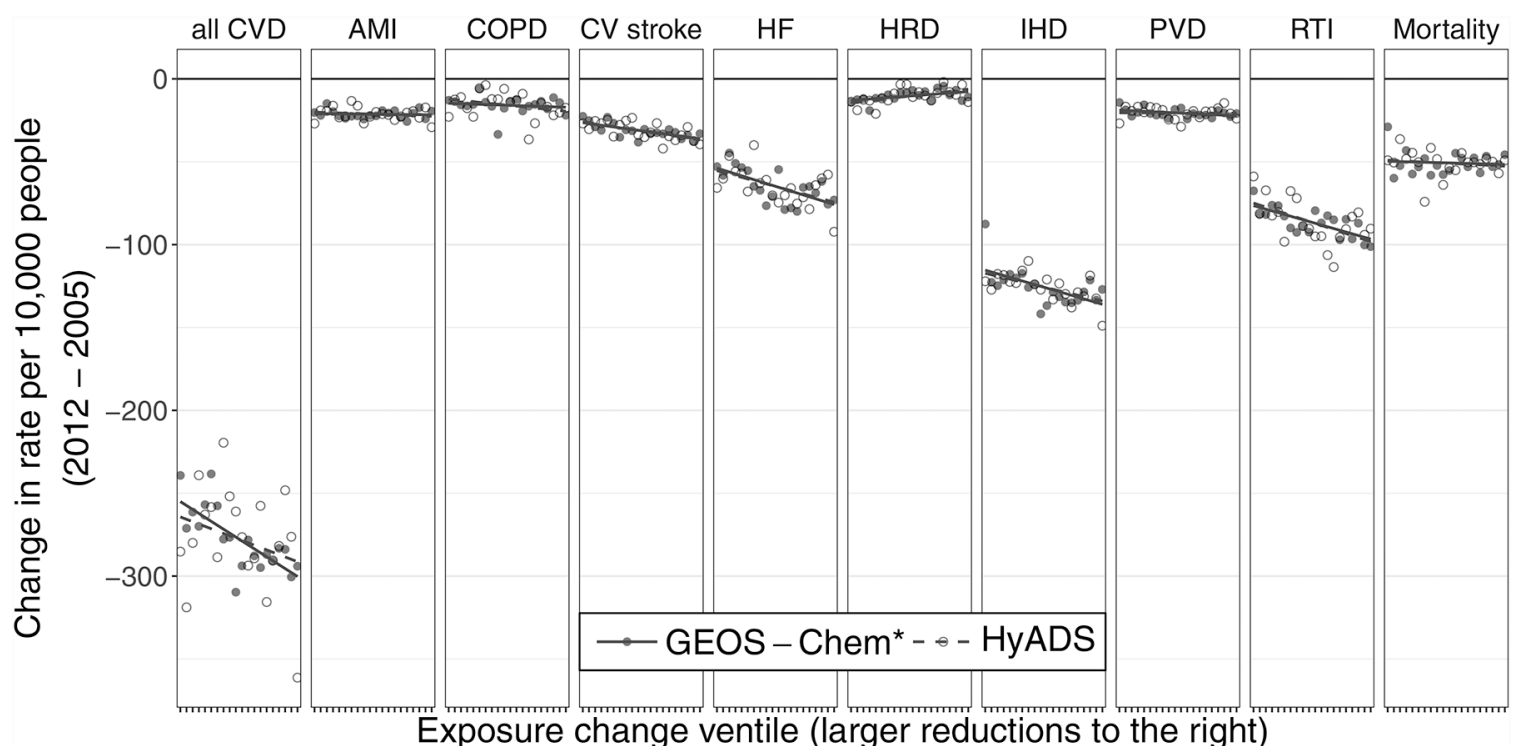

FIGURE 3.

Changes in hospitalization rate per 10,000 person-years (beneficiaries for all Mortality) in ZIP codes that fall in each exposure change ventile $\left(-\beta_{q}\right)$ estimated with model 2 . Negative values on the vertical axis denote decreases in health outcome rates. CV, cardiovascular; HF, heart failure; HRD, heart rhythm disorders. 
Table 1.

Mean (interquartile range width) exposure metrics $(E)$ in ZIP codes across the United States east of -110 longitude in 2005, 2012, and differences between years (negative denotes decrease)

\begin{tabular}{|l|l|l|l|}
\hline & $\boldsymbol{E}_{\mathbf{2 0 0 5}}$ & $\boldsymbol{E}_{\mathbf{2 0 1 2}}$ & $E_{2012-2005}^{\Delta}$ \\
\hline $\mathrm{PM}_{2.5}^{\operatorname{sim}\left(\mu \mathrm{gm}^{-3}\right)}$ & $10.0(4.5)$ & $7.2(2.6)$ & $-3.2(2.4)$ \\
\hline HyADS $^{\text {adj }}\left(\mu \mathrm{gm}^{-3 *}\right)$ & $2.4(1.6)$ & $1.20(0.6)$ & $-1.2(1.1)$ \\
\hline HyADS (unitless) & $2.9 \times 10^{5}\left(3.0 \times 10^{5}\right)$ & $8.2 \times 10^{4}\left(6.6 \times 10^{4}\right)$ & $-2.0 \times 10^{5}\left(2.4 \times 10^{5}\right)$ \\
\hline
\end{tabular}


Table 2.

Mean (interquartile range width) hospitalization rates per 10,000 person-years (beneficiaries for all Mortality; $R$ ) in ZIP codes east of -110 longitude in 2005 and 2012, and differences between years (negative denotes decrease)

\begin{tabular}{|l|l|l|l|}
\hline & $\boldsymbol{R}_{\mathbf{2 0 0 5}}$ & $\boldsymbol{R}_{\mathbf{2 0 1 2}}$ & $R_{2012-2005}^{\Delta}$ \\
\hline All CVD & $1000(500)$ & $760(380)$ & $-280(410)$ \\
\hline AMI & $140(80)$ & $110(70)$ & $-20(70)$ \\
\hline COPD & $170(120)$ & $150(110)$ & $-15(90)$ \\
\hline CV stroke & $210(110)$ & $170(90)$ & $-31(100)$ \\
\hline HF & $260(170)$ & $190(130)$ & $-64(140)$ \\
\hline HRD & $180(90)$ & $160(90)$ & $-10(100)$ \\
\hline IHD & $330(180)$ & $200(120)$ & $-120(150)$ \\
\hline PVD & $98(60)$ & $72(40)$ & $-20(50)$ \\
\hline RTI & $280(170)$ & $190(120)$ & $-86(140)$ \\
\hline All mortality & $510(200)$ & $460(180)$ & $-52(210)$ \\
\hline
\end{tabular}

$\mathrm{CV}$, cardiovascular; HF, heart failure; HRD, heart rhythm disorders. 